\title{
Coexpression Analysis of Transcriptome on AIDS and Other Human Disease Pathways by Canonical Correlation Analysis
}

\author{
Yahong Chen, ${ }^{1,2}$ Jinjin Yuan, ${ }^{1,2}$ Xianlin Han, ${ }^{3}$ Xiaolong Liu, ${ }^{1,2}$ Xiao Han, ${ }^{4}$ and Hanhui Ye ${ }^{1,2}$ \\ ${ }^{1}$ The United Innovation of Mengchao Hepatobiliary Technology Key Laboratory of Fujian Province, Mengchao Hepatobiliary \\ Hospital of Fujian Medical University, Fuzhou 350025, China \\ ${ }^{2}$ Infectious Diseases Hospital of Fuzhou, Fuzhou 350025, China \\ ${ }^{3}$ Department of General Surgery, Peking Union Medical College Hospital, Beijing, China \\ ${ }^{4}$ Biotechnology Research Institute, Chinese Academy of Agricultural Sciences, Beijing 100081, China \\ Correspondence should be addressed to Xiao Han; hanxiao@caas.cn and Hanhui Ye; yehanhui@163.com
}

Received 22 September 2016; Revised 24 February 2017; Accepted 8 March 2017; Published 14 June 2017

Academic Editor: Mohamed Salem

Copyright (C) 2017 Yahong Chen et al. This is an open access article distributed under the Creative Commons Attribution License, which permits unrestricted use, distribution, and reproduction in any medium, provided the original work is properly cited.

\begin{abstract}
Acquired immune deficiency syndrome is a severe disease in humans caused by human immunodeficiency virus. Several human genes were characterized as host genetic factors that impact the processes of AIDS disease. Recent studies on AIDS patients revealed a series disease is complicating with AIDS. To resolve gene interaction between AIDS and complicating diseases, a canonical correlation analysis was used to identify the global correlation between AIDS and other disease pathway genes expression. The results showed that HLA-B, HLA-A, MH9, ZNED1, IRF1, TLR8, TSG101, NCOR2, and GML are the key AIDS-restricted genes highly correlated with other disease pathway genes. Furthermore, pathway genes in several diseases such as asthma, autoimmune thyroid disease, and malaria were globally correlated with ARGs. It suggests that these diseases are a high risk in AIDS patients as complicating diseases.
\end{abstract}

\section{Introduction}

Human immunodeficiency virus (HIV) causes a serious disease that affects people's health and lives. Millions of people have died from HIV infections in the 30 years since its identification. Over the past decades, a large number of studies have focused on every aspect of this virus, including virology, immunology, treatment, and genetics. An important problem related to AIDS complications was raised after the discovery of the HIV characteristics that severely damaged the lymphoid system.

Several diseases are associated with HIV infection and antiretroviral therapy. Tuberculosis is highly frequent in a large proportion of HIV infection cases in developing countries [1]. When research revealed that HIV-induced immune deficiency was the most common risk factor for cancer, HIV infection-related cancer became a complication of HIV infection [2]. HIV-associated sensory neuropathy is also a complication of HIV infection [3]. Recently, venous thrombosis has been described as a disease associated with HIV-positive patients [4]. Pulmonary arterial hypertension is a life-threatening complication of HIV infection [5]. A well-described complication of HIV and antiretroviral therapy is pancreatitis, which has exceedingly high rates in the HIV-positive population [6]. During antiretroviral therapy, classical Hodgkin lymphoma (HIV-cHL) and rhabdomyolysis are also important complications of HIV disease $[7,8]$. One report showed that HIV patients frequently had neutropenia [9]. Generally, AIDS complications are involved in almost all important human diseases to the best of our knowledge.

Our understanding of genetic restriction factors targeting AIDS has been greatly improved by advances in genome research, such as sequencing of the whole human genome through physical and functional analyses. Many methods have been developed to study the underlying mechanisms of diseases on the whole genome level, such as genomewide association studies, which can identify host genetic 
factors that affect HIV infection and the host restriction response. Nearly 40 AIDS restriction genes (ARGs) were identified from widely biological pathways such as the HIV entry receptor on lymphoid cells to oncogenes in human glioblastomas.

Many web-based databases, such as KEGG, have been established as tools to collect human disease pathway genes using genomic and molecular methods. For example, comparative transcriptome analysis can isolate marker genes that are highly differentially expressed in patients. Molecular biology has discovered several pathways that play major roles in human diseases, and hundreds of genes have been characterized as members of human disease pathways. Hence, human disease genes are available for the analysis of their effects on ARGs.

Expression correlation between genes based on a gene coexpression model can reveal the molecular mechanisms underlying gene regulation. For instance, mitochondrial pathways are coexpressed with muscle system pathway genes and neurodegenerative disease pathway genes [10]. The expression correlation between ARGs and other disease pathways could also explain the relationship between ARGs and AIDS complicating diseases. Canonical correlation analysis (CCA) is a powerful approach to detect coexpression between gene sets because it does not only determine correlations between two genes. For example, CCA can be used to perform a coexpression analysis of glioma pathway genes from glioblastoma transcriptomes.

In this study, we developed a CCA to determine coexpression between ARGs and other human disease pathway genes. We discussed the most significant coexpression patterns that could imply the susceptibility or sensitization to AIDS complicating diseases.

\section{Methods}

2.1. Datasets. Expression data on 20,000 human genes in human genome at about 4000 experiments was normalized to a human genome expression datasets hsa.v14-04.G19816S5626 were downloaded from COPRESDB (http://coxpresdb. $\mathrm{jp} /$ ). It includes many experiments not only related to HIV infection. Human disease pathway genes (br08402.keg updated 2016) were downloaded from KEGG (http://www. keg.jp); this dataset includes 68 typical disease pathways with key genes (Table 1). From published literature, we collected about 39 ARGs (see Table S1 available online at https://doi.org/10.1155/2017/9163719). From human genome expression datasets, two expression datasets were generated to include disease pathway genes and ARG expression data, respectively.

2.2. Canonical Correlation Analysis. Canonical correlation analysis is a statistical method which extracts statistically independent pair of canonical variables dependent on the correlation among two sets of original variables. The original variables are results of a linear combination of the canonical variables. In this study, expression of disease pathway genes in diverse conditions was described by vector $a=\left(a_{1}, a_{2}, \ldots, a_{m}\right)$ and ARG expression by the vector $b=\left(b_{1}, b_{2}, \ldots, b_{m}\right)$. The respective canonical variables $c=\left(c_{1}, c_{2}, \ldots, c_{m}\right)$ and $d=\left(d_{1}, d_{2}, \ldots, d_{m}\right)$ have canonical coefficients vectors $s=\left(s_{1}, s_{2}, \ldots, s_{m}\right)$ and $s^{\prime}=\left(s^{\prime}{ }_{1}, s^{\prime}{ }_{2}, \ldots, s^{\prime}{ }_{m}\right)$. $a=c^{\prime}$ s, and $b=d^{\prime} \mathrm{s}^{\prime}$. The vector of eigenvalues was calculated as the magnitude of the correlation between pair of canonical variables. The variance covariance matrices were used to analyze the covariances between variables.

2.3. The Study Design and Software Tools. The R platform (http://www.rproject.org/) was used for canonical correlation analysis of expression data. After the canonical variables were produced, the top correlated canonical variables $(r>0.95)$ were identified to analysis the coexpressed individual genes. Two thresholds were set up to isolate correlated integrated disease pathways with $r$ values $>0.5$ and standard deviations $>0.2$. Web-based DAVID tool (http:// david.abcc.ncifcrf.gov/) was used for functional annotations and enrichment analysis; we used Homo sapiens genome as background. The "KEGG_PATHWAY" was selected for disease pathway enrichment analysis. Other parameters were automatically generated from DAVID.

Functional annotations were generated, and enrichment analyses were performed for the metabolic pathway genes using the web-based DAVID tool (http://david.abcc. ncifcrf.gov/). For the pathway enrichment analyses, the "KEGG_PATHWAY" was selected. The pathways with a $P$ value $<0.01$ were considered significant.

\section{Results and Discussion}

3.1. ARGs. Nearly 40 AIDS restriction genes (ARGs) have been considered as host genes that impact the progression of HIV infection from virus entry to the development of AIDS (Table S1). For example, PPIA, TSG101, TRIM5 $\alpha$, APOBEC3G, and CUL5 encode HIV-1 postentry cellular viral cofactors that have been described in recent research. PPIA plays a role in cyclosporin A-mediated immunosuppression as a member of the peptidyl-prolyl cis-trans isomerase (PPIase) family [11], which can interact with HIV viral proteins. Cell growth and differentiation are regulated by the interaction of TSG101 with stathmin [12]. TRIM5 $\alpha$ is an E3 ubiquitin ligase that is involved in retroviral restriction [13]. Other ARGs are involved in many cellular processes, such as DEFB1, which has been implicated in cystic fibrosis pathogenesis [14], HLA-A, which is expressed in nearly all cells [15], CCL, which has been implicated in immunoregulatory and inflammatory processes [16], CXCR6, which is a chemokine (C-X-C motif) receptor [17], LY6D, which is a member of the lymphocyte antigen 6 complex [18], and APOBEC3B, which is a cytidine deaminase. However, these ARGs have been characterized only in the absence of AIDS-related complications. The relationship between ARGs and other human diseases is unknown.

3.2. The General CCA Results. The application of CCA to a transcriptome can identify coexpression between genes. Coexpression between individual genes or groups of genes can be identified based on the standard deviations of genes on canonical variables. Hence, we used two strategies to 
TABLE 1: Human disease pathway in KEGG.

\begin{tabular}{|c|c|c|}
\hline Classes & ID & Pathways \\
\hline \multirow{6}{*}{ Cancers: overview } & 5200 & Pathways in cancer \\
\hline & 5202 & $\begin{array}{c}\text { Transcriptional misregulation in } \\
\text { cancer }\end{array}$ \\
\hline & 5203 & Viral carcinogenesis \\
\hline & 5204 & Chemical carcinogenesis \\
\hline & 5205 & Proteoglycans in cancer \\
\hline & 5206 & MicroRNAs in cancer \\
\hline \multirow{14}{*}{ Cancers: specific types } & 5210 & Colorectal cancer \\
\hline & 5211 & Renal cell carcinoma \\
\hline & 5212 & Pancreatic cancer \\
\hline & 5213 & Endometrial cancer \\
\hline & 5214 & Glioma \\
\hline & 5215 & Prostate cancer \\
\hline & 5216 & Thyroid cancer \\
\hline & 5217 & Basal cell carcinoma \\
\hline & 5218 & Melanoma \\
\hline & 5219 & Bladder cancer \\
\hline & 5220 & Chronic myeloid leukemia \\
\hline & 5221 & Acute myeloid leukemia \\
\hline & 5222 & Small cell lung cancer \\
\hline & 5223 & Non-small-cell lung cancer \\
\hline \multirow{8}{*}{ Immune diseases } & 5310 & Asthma \\
\hline & 5320 & Autoimmune thyroid disease \\
\hline & 5321 & Inflammatory bowel disease (IBD) \\
\hline & 5322 & Systemic lupus erythematosus \\
\hline & 5323 & Rheumatoid arthritis \\
\hline & 5330 & Allograft rejection \\
\hline & 5332 & Graft-versus-host disease \\
\hline & 5340 & Primary immunodeficiency \\
\hline \multirow{5}{*}{$\begin{array}{l}\text { Neurodegenerative } \\
\text { diseases }\end{array}$} & 5010 & Alzheimer's disease \\
\hline & 5012 & Parkinson's disease \\
\hline & 5014 & Amyotrophic lateral sclerosis (ALS) \\
\hline & 5016 & Huntington's disease \\
\hline & 5020 & Prion diseases \\
\hline \multirow{5}{*}{$\begin{array}{l}\text { Substance } \\
\text { dependence }\end{array}$} & 5030 & Cocaine addiction \\
\hline & 5031 & Amphetamine addiction \\
\hline & 5032 & Morphine addiction \\
\hline & 5033 & Nicotine addiction \\
\hline & 5034 & Alcoholism \\
\hline \multirow{4}{*}{$\begin{array}{l}\text { Cardiovascular } \\
\text { diseases }\end{array}$} & 5410 & $\begin{array}{l}\text { Hypertrophic cardiomyopathy } \\
\text { (HCM) }\end{array}$ \\
\hline & 5412 & $\begin{array}{l}\text { Arrhythmogenic right ventricular } \\
\text { cardiomyopathy (ARVC) }\end{array}$ \\
\hline & 5414 & Dilated cardiomyopathy \\
\hline & 5416 & Viral myocarditis \\
\hline \multirow{3}{*}{$\begin{array}{l}\text { Endocrine and } \\
\text { metabolic diseases }\end{array}$} & 4930 & Type II diabetes mellitus \\
\hline & 4940 & Type I diabetes mellitus \\
\hline & 4950 & Maturity onset diabetes of the young \\
\hline
\end{tabular}

TABle 1: Continued.

\begin{tabular}{|c|c|c|}
\hline Classes & ID & Pathways \\
\hline \multirow{10}{*}{$\begin{array}{l}\text { Infectious diseases: } \\
\text { bacterial }\end{array}$} & 5100 & Bacterial invasion of epithelial cells \\
\hline & 5110 & Vibrio cholerae infection \\
\hline & 5120 & $\begin{array}{l}\text { Epithelial cell signaling in } \\
\text { Helicobacter pylori infection }\end{array}$ \\
\hline & 5130 & Pathogenic Escherichia coli infection \\
\hline & 5131 & Shigellosis \\
\hline & 5132 & Salmonella infection \\
\hline & 5133 & Pertussis \\
\hline & 5134 & Legionellosis \\
\hline & 5150 & Staphylococcus aureus infection \\
\hline & 5152 & Tuberculosis \\
\hline \multirow{7}{*}{$\begin{array}{l}\text { Infectious diseases: } \\
\text { viral }\end{array}$} & 5160 & Hepatitis C \\
\hline & 5161 & Hepatitis B \\
\hline & 5162 & Measles \\
\hline & 5164 & Influenza A \\
\hline & 5166 & HTLV-I infection \\
\hline & 5168 & Herpes simplex infection \\
\hline & 5169 & Epstein-Barr virus infection \\
\hline \multirow{6}{*}{$\begin{array}{l}\text { Infectious diseases: } \\
\text { parasitic }\end{array}$} & 5140 & Leishmaniasis \\
\hline & 5142 & $\begin{array}{l}\text { Chagas disease (American } \\
\text { trypanosomiasis) }\end{array}$ \\
\hline & 5143 & African trypanosomiasis \\
\hline & 5144 & Malaria \\
\hline & 5145 & Toxoplasmosis \\
\hline & 5146 & Amoebiasis \\
\hline
\end{tabular}

determine two types of correlation. First, the top $(r>0.95)$ canonical correlations with low standard deviations were isolated as coexpressed individual genes. Coexpression has been suggested to have less of an impact on whole disease pathways. The relationship between complicating diseases and AIDS is dependent on the roles of a few genes. Second, canonical correlations with high standard deviations $(s>0.2)$ and $r$ values $(>0.5)$ were selected as coexpressed between gene groups. This result indicates that coexpression has an effect on the whole disease pathway because more than $20 \%$ of the genes contribute to the canonical correlation. Most genes in disease pathways are involved in the cross talk between the complicating disease and AIDS.

3.3. Coexpressed ARGs and Human Disease Genes. As shown in Table 2, 21 top $(r>0.95)$ canonical correlations were determined between the ARGs and human disease pathway gene transcriptomes using the CCA approach. The canonical variables originated from disease pathways including HTLV-I infection, herpes simplex infection, Epstein-Barr virus infection, viral carcinogenesis, viral myocarditis, type I diabetes mellitus, graft-versus-host disease, autoimmune thyroid disease, allograft rejection, pathways in cancer, influenza $\mathrm{A}$, proteoglycans in cancer, tuberculosis, transcriptional misregulation in cancer, Huntington's disease, toxoplasmosis, hepatitis B, measles, microRNAs in cancer, hepatitis $C$, and Alzheimer's disease. However, canonical variables could 
TABLE 2: Typical disease pathways have high coexpression with ARGs. $r$ is the correlation factor; Sa, standard deviation of canonical variables in a (ARGs); Sb, standard deviation of canonical variables in b (disease pathways); Wilks, Wilks' lambda (likelihood ratio) statistic; and Chisq, Bartlett's approximate chi-squared statistic for $\mathrm{H}(\mathrm{k}) 0$ with Lawley's modification.

\begin{tabular}{|c|c|c|c|c|c|c|}
\hline Pathway ID & Pathways & $r$ & $\mathrm{Sa}$ & $\mathrm{Sb}$ & Wilks & Chisq \\
\hline 5166 & HTLV-I infection & 0.988 & 0.0064 & 0.0119 & $5.56 E-14$ & 129868.5 \\
\hline 5168 & Herpes simplex infection & 0.987 & 0.0064 & 0.0112 & $1.22 E-11$ & 107961 \\
\hline 5169 & Epstein-Barr virus infection & 0.987 & 0.0065 & 0.0120 & $4.46 E-12$ & 112040.6 \\
\hline 5203 & Viral carcinogenesis & 0.987 & 0.0068 & 0.0126 & $1.05 E-11$ & 108466.7 \\
\hline 5416 & Viral myocarditis & 0.983 & 0.0073 & 0.0130 & $1.24 E-07$ & 69145.74 \\
\hline 4940 & Type I diabetes mellitus & 0.982 & 0.0078 & 0.0145 & $8.94 E-06$ & 50709.06 \\
\hline 5332 & Graft-versus-host disease & 0.981 & 0.0080 & 0.0141 & $2.50 E-05$ & 46246.41 \\
\hline 5320 & Autoimmune thyroid disease & 0.981 & 0.0083 & 0.0149 & $2.52 E-05$ & 46159.16 \\
\hline 5330 & Allograft rejection & 0.980 & 0.0081 & 0.0146 & $6.08 E-05$ & 42385.9 \\
\hline 5200 & Pathways in cancer & 0.972 & 0.0091 & 0.0103 & $1.39 E-14$ & 134581 \\
\hline 5164 & Influenza A & 0.964 & 0.0079 & 0.0086 & $7.45 E-11$ & 100289.5 \\
\hline 5205 & Proteoglycans in cancer & 0.964 & 0.0116 & 0.0088 & $4.19 E-12$ & 111889.7 \\
\hline 5152 & Tuberculosis & 0.960 & 0.0083 & 0.0088 & $6.84 E-11$ & 100606.8 \\
\hline 5202 & Transcriptional misregulation in cancer & 0.959 & 0.0107 & 0.0097 & $9.35 E-11$ & 99265.29 \\
\hline 5016 & Huntington's disease & 0.958 & 0.0209 & 0.0162 & $1.09 E-09$ & 88602.38 \\
\hline 5145 & Toxoplasmosis & 0.958 & 0.0093 & 0.0089 & $8.41 E-09$ & 80437.34 \\
\hline 5161 & Hepatitis B & 0.957 & 0.0117 & 0.0101 & $6.25 E-10$ & 91320.55 \\
\hline 5162 & Measles & 0.956 & 0.0098 & 0.0105 & $1.27 E-09$ & 88462.56 \\
\hline 5206 & MicroRNAs in cancer & 0.956 & 0.0131 & 0.0093 & $4.63 E-10$ & 92549.92 \\
\hline 5160 & Hepatitis C & 0.952 & 0.0129 & 0.0111 & $3.99 E-09$ & 83502.52 \\
\hline 5010 & Alzheimer's disease & 0.951 & 0.0192 & 0.0153 & $1.18 E-09$ & 88451.59 \\
\hline
\end{tabular}

not delegate total ARG expression and human disease pathway gene expression because the standard deviations of the ARGs (Sa) and the human disease pathway genes ( $\mathrm{Sb}$ ) were less than 0.05, as shown in Table 2. Sa and Sb indicate that the canonical variables can explain less than $5 \%$ of the genes among the ARGs and human disease pathway genes. For example, HTLV-I infection pathway has 0.988 correlation factor with ARGs, and Wilks and Chisq indicate the statistic of correlation. But it has $\mathrm{Sa}<0.01$ and $\mathrm{Sb}<0.05$, which suggested that in whole pathway of HTLV-I infection, only a few genes were correlated with ARGs.

In Table 3, the genes with the highest correlation with the canonical variables from the ARGs and human disease pathway genes were collected to show the coexpression relationships.

3.3.1. HLA-B and HLA-A. HLA-B and HLA-A are MHC class I molecules that strongly impact HIV-1 progression (Table 3) [19]. HLA polymorphisms are significantly correlated with the time to AIDS in HIV-infected individuals [20]. HLA-A and HLA-B are major AIDS restriction genes.

HLA-B and HLA-B were coexpressed with 3135 (HLA-G major histocompatibility complex, class I, G) and 3134 (HLA-F major histocompatibility complex, class I, F) genes shared by many disease pathways, including HTLV-I infection, herpes simplex infection, Epstein-Barr virus infection, viral carcinogenesis, viral myocarditis, type I diabetes mellitus, graft-versus-host disease, autoimmune thyroid disease, and allograft rejection (Table 3). As a marker of $\mathrm{T}$ cell activation, HLA-DR induction was associated with HTLV-I seropositivity [21]. HTLV-I infection leads to the induction of HLA [22]. Herpes simplex virus type 1 (HSV1) can upregulate HLA-G expression in human neurons after acute neuron infection [23], whereas HLA-G is the MHC class I molecule that is induced in B cells after Epstein-Barr virus transformation [24]. During viral carcinogenesis, HLA is abnormally expressed to enable cancer cells to escape from immune surveillance [25]. HLA-G polymorphisms and expression were suggested as diagnostic markers due to their involvement in breast carcinogenesis [26]. The increased occurrence of HLA antigens was shown to be associated with viral myocarditis [27]. The HLA complex has been reported to contribute to type 1 diabetes because HLA polymorphisms introduce genetic susceptibility to type 1 diabetes [28, 29]. There is evidence that HLA gene polymorphisms are potent risk factors for severe acute graft versus host diseases [30]. It was also shown that an HLA variant conferred a high risk for autoimmune thyroid disease [31]. Furthermore, allelicinduced abnormal expression of the HLA-G gene has been suggested to be associated with acute allograft rejection [32]. Generally, coexpression of the HLA genes indicates cross talk between HIV infection and other diseases, and this cross talk provides a potent mechanism for these diseases becoming AIDS complicating diseases.

3.3.2. MYH9 and ZNRD1. MYH9 encodes a conventional nonmuscle myosin that is downregulated by HIV-1 pathogenesis [33]. As a zinc ribbon domain-containing protein, 
TABLE 3: AIDS-restricted genes have high coexpression with other diseases.

\begin{tabular}{|c|c|c|c|}
\hline ID & Pathways & ARGs & Pathway genes \\
\hline 5166 & HTLV-I infection & HLA-B, HLA-A & 3135,3134 \\
\hline 5168 & Herpes simplex infection & HLA-B, HLA-A & 3135,3134 \\
\hline 5169 & Epstein-Barr virus infection & HLA-B, HLA-A & 3135,3134 \\
\hline 5203 & Viral carcinogenesis & HLA-B, HLA-A & 3135,3134 \\
\hline 5416 & Viral myocarditis & HLA-B, HLA-A & 3135,3134 \\
\hline 4940 & Type I diabetes mellitus & HLA-B, HLA-A & 3135,3134 \\
\hline 5332 & Graft-versus-host disease & HLA-B, HLA-A & 3135,3134 \\
\hline 5320 & Autoimmune thyroid disease & HLA-B, HLA-A & 3135,3134 \\
\hline 5330 & Allograft rejection & HLA-B, HLA-A & 3135,3134 \\
\hline 5200 & Pathways in cancer & MYH9, ZNRD1 & 7428,6772 \\
\hline 5164 & Influenza A & IRF1, ZNRD1 & 356,5609 \\
\hline 5205 & Proteoglycans in cancer & MYH9, ZNRD1 & 5781,5293 \\
\hline 5152 & Tuberculosis & TLR8, ZNRD1 & 6772,3587 \\
\hline 5202 & Transcriptional misregulation in cancer & ZNRD1, MYH9 & 5371,51513 \\
\hline 5016 & Huntington's disease & ZNRD1, TSG101 & 25981,55567 \\
\hline 5145 & Toxoplasmosis & MYH9, ZNRD1 & 2775,4261 \\
\hline 5161 & Hepatitis B & MYH9, ZNRD1 & 5111,356 \\
\hline 5162 & Measles & NCOR2, ZNRD1 & 356,6772 \\
\hline 5206 & MicroRNAs in cancer & MYH9, ZNRD1 & 7143,6655 \\
\hline 5160 & Hepatitis C & ZNRD1, GML & 6772,6655 \\
\hline 5010 & Alzheimer's disease & NCOR2, ZNRD1 & 4035,9377 \\
\hline
\end{tabular}

ZNRD1 plays a role in HIV-1 replication and AIDS progression [34]. A recent study found that ZNRD1 polymorphisms had a significant influence on AIDS progression [35]. ZNRD1 and MYH9 are major AIDS restriction genes.

Table 3 shows that MYH9 and ZNRD1 are coexpressed with different genes in disease pathways, such as 7428 (VHL; von Hippel-Lindau tumor suppressor) and 6772 (STAT1; signal transducer and activator of transcription 1) in cancer, 5781 (PTPN11; protein tyrosine phosphatase, nonreceptor type 11) and 5293 (PIK3CD; phosphatidylinositol-4,5bisphosphate 3-kinase catalytic subunit delta) in proteoglycans in cancer, 5371 (PML; promyelocytic leukemia) and 51513 (ETV7; ETS variant 7) in transcriptional misregulation in cancer, 7143 (TNR; tenascin R) and 6655 (SOS2; SOS Ras/Rho guanine nucleotide exchange factor 2) in microRNAs in cancer, 2775 (GNAO1; G protein subunit alpha o1) and 4261 (CIITA; class II, major histocompatibility complex, transactivator) in toxoplasmosis, and 5111 (PCNA; proliferating cell nuclear antigen) and 356 (FASLG; Fas ligand) in hepatitis $\mathrm{B}$.

VHL is a well-known tumor suppressor that is involved in hereditary cancer syndromes [36]. Inhibition of STAT1 can block cancer cell proliferation and invasion [37]. Overexpression of PTPN11 can enhance the progression of many proteoglycan cancers, such as liver cancer [38]. Inhibition of PIK3CD signaling can abrogate transitions in cancer cells [39]. Downregulation of PML, which is involved in the cell cycle, survival, and apoptosis, was identified in cancer cells [40]. ETV7 plays important roles in chromosomal translocations in human cancer [41]. Induction of SOS2 was demonstrated in cancer cells, such as triple negative breast cancer [42]. Toxoplasma gondii can downregulate CIITA to inhibit MHC class II expression [43]. The PCNA promoter can recruit the hepatitis $B$ virus preS protein to become active [44]. Additionally, the Fas ligand gene can be induced by the hepatitis B virus X protein [45]. Thus, the coexpression of MYH9, ZNRD1, and other disease pathway genes suggests probable AIDS complicating diseases.

3.3.3. IRF1 and ZNRD1. IRF1 is an interferon regulatory factor that can affect productive HIV infection and support natural resistance against HIV infection [46]. Hence, IRF1 is a well-studied human AIDS restriction gene.

As shown in Table 3, IRF1 and ZNRD1 are coexpressed with 356 (FASLG; Fas ligand) and 5609 (MAP2K7; mitogen-activated protein kinase 7) in the influenza A pathways. FASLG is described as a hepatitis B pathway gene and plays an important function in the influenza A virus pathway because influenza virus infection induces Fas ligand expression when the infected cells contact one another [47].

3.3.4. TLR8 and ZNRD1. TLR8 is a human toll-like receptor that plays a role in signaling pathways that modulate the innate immune response to HIV infection and reduce HIV replication [48]. Genetic polymorphisms in TLR8 have been determined to be host cell factors associated with HIV status [49].

As shown in Table 3, TLR8 and ZNRD1 are coexpressed with 6772 (STAT1; signal transducer and activator of transcription 1) and 3587 (IL10RA; interleukin 10 receptor 
TABLE 4: Canonical correlation between integrated disease pathway genes and ARGs.

\begin{tabular}{|c|c|c|c|c|c|}
\hline ID & Pathways & Pair ID & $\mathrm{Sa}$ & $\mathrm{Sb}$ & $r$ \\
\hline 5211 & Renal cell carcinoma & 14 & 0.26 & 0.21 & 0.5 \\
\hline 5213 & Endometrial cancer & 12 & 0.26 & 0.21 & 0.51 \\
\hline 5219 & Bladder cancer & 11 & 0.29 & 0.22 & 0.5 \\
\hline 5221 & Acute myeloid leukemia & 12 & 0.37 & 0.21 & 0.52 \\
\hline 5223 & Non-small-cell lung cancer & 8 & 0.23 & 0.21 & 0.62 \\
\hline 5310 & Asthma & 4 & 0.22 & 0.42 & 0.66 \\
\hline 5320 & Autoimmune thyroid disease & 7 & 0.27 & 0.4 & 0.57 \\
\hline 5330 & Allograft rejection & 7 & 0.31 & 0.26 & 0.52 \\
\hline 5332 & Graft-versus-host disease & 4 & 0.21 & 0.23 & 0.72 \\
\hline 5340 & Primary immunodeficiency & 8 & 0.22 & 0.24 & 0.55 \\
\hline 5033 & Nicotine addiction & 3 & 0.35 & 0.41 & 0.58 \\
\hline 4940 & Type I diabetes mellitus & 6 & 0.24 & 0.24 & 0.64 \\
\hline 5100 & Bacterial invasion of epithelial cells & 4 & 0.21 & 0.49 & 0.62 \\
\hline 5130 & Pathogenic Escherichia coli infection & 12 & 0.25 & 0.21 & 0.58 \\
\hline 5144 & Malaria & 8 & 0.24 & 0.21 & 0.54 \\
\hline
\end{tabular}

subunit alpha) in the tuberculosis pathways. STAT1 plays a role in a signaling pathway to control intracellular killing of phagocytosed Mycobacterium tuberculosis [50]. IL-10 is a key factor that mediates the immunopathogenesis of tuberculosis in combination with interferon gamma and adiponectin [51].

3.3.5. TSG101 and ZNRD1. TSG101 interplays with the HIV-1 nucleocapsid to prohibit HIV from turning into a DNA-containing virus [52]. Therefore, TSG101 is a typical anti-HIV drug target and human AIDS restriction gene [53].

As shown in Table 3, TSG101 and ZNRD1 are coexpressed with 25981 (DNAH1; dynein axonemal heavy chain 1) and 55567 (DNAH3; dynein axonemal heavy chain 3 ) in the Huntington's disease pathways.

3.3.6. NCOR2 and ZNRD1. NCOR2 is the RING finger ubiquitin ligase and nuclear corepressor that downregulates HIV1 replication [54]. NCOR2 is a major AIDS restriction gene.

As shown in Table 3, NCOR2 and ZNRD1 are coexpressed with 356 (FASLG; Fas ligand) and 6772 (STAT1; signal transducer and activator of transcription 1) in the measles pathways. Fas ligand and STAT1 were previously described in other disease pathways. Moreover, the measles virus phosphoprotein can prevent STAT1 phosphorylation [55].

As shown in Table 3, NCOR2 and ZNRD1 are coexpressed with 4035 (LRP1; LDL receptor-related protein 1) and 9377 (COX5A; cytochrome c oxidase subunit 5A) in the Alzheimer's disease pathways. Recent studies showed that the LRP1 levels were reduced in Alzheimer's disease [56]. COX family genes can impair anxiety-like behavior in an Alzheimer's disease model [57].

3.3.7. GML and ZNRD1. GML is the LY6 family member of the glycosylphosphatidylinositol- (GPI-) anchored proteins, which have conserved cysteine-rich domains with specific disulfide bonding patterns. LY6H was upregulated by HIV infection and suggested to play a role in innate immunity to HIV-1 via an interferon-like mechanism. Gene polymorphism analysis results support the hypothesis that GML is a susceptibility locus for HIV-1 infection [58].

GML and ZNRD1 are coexpressed with 6772 (STAT1; signal transducer and activator of transcription 1) and 6655 (SOS2; SOS Ras/Rho guanine nucleotide exchange factor 2) in the hepatitis $\mathrm{C}$ pathways. STAT1 plays an important role in hepatitis $\mathrm{C}$ infection in addition to cancer, tuberculosis, and measles. For example, hepatic STAT1 undergoes nuclear translocation during hepatitis $C$ virus infection [59]. SOS2 has major functions in hepatitis $\mathrm{C}$ disease and cancer.

3.4. Coexpressed ARGs and Human Disease Pathways. As shown in Table 4, canonical correlations with large $\mathrm{Sa}, \mathrm{Sb}$ $(>0.2)$, and $r$ values $(>0.5)$ were selected to analyze coexpression between integral disease pathways and global ARGs. Unlike the top canonical correlations $(r>0.95)$ shown in Table 2, these canonical variables showed less correlation between each disease pathway and the ARG (Table 4). The maximum $r$ was detected between the asthma pathways and ARGs (equal to 0.66). However, these canonical variables had larger standard deviations (at least 0.2) than the top canonical correlations described previously. In the maximum case, the Sa of the ARG correlated with the acute myeloid leukemia pathway was 0.37 and the Sb of the ARG correlated with the bacterial invasion of epithelial cells pathway was 0.47. Large standard deviations indicate that more ARGs or disease pathway genes are involved in the canonical variable. Additionally, these canonical correlations show a certain level of Pearson correlation, which determines coexpression. This result suggests that most of the genes in disease pathways are coexpressed with most of the ARGs at the whole pathway level. For example, renal cell carcinoma pathway showed coexpression with ARGs with $r=0.5, \mathrm{Sa}=0.26$, and $\mathrm{Sb}=0.21$. It indicates that $26 \% \mathrm{ARG}$ genes were 
expressed with $21 \%$ renal cell carcinoma pathway genes. Rather than find a single gene correlated with ARGs, CCA could identify a group of genes in certain pathways correlated with ARGs. This indicate more significant and higher potential than a single gene. These pathways include renal cell carcinoma, endometrial cancer, bladder cancer, acute myeloid leukemia, non-small-cell lung cancer, asthma, autoimmune thyroid disease, allograft rejection, graft-versus-host disease, primary immunodeficiency, nicotine addiction, type I diabetes mellitus, bacterial invasion of epithelial cells, pathogenic Escherichia coli infection, and malaria.

The coexpression identified from CCA is preferred to describe a general relationship since the transcriptome datasets include not only HIV-related experiments but also diverse biological experiments. Due to no limitation of samples in datasets, diseases in other human organs could be characterized as coexpressed pathway with AIDS. This provide a possibility to establish a precise experiment to determine this relationship.

Among these diseases, asthma is prevalent in populations with HIV infections because $20 \%$ of individuals infected with HIV have asthma in some clinical investigations [60]. There is evidence to support the hypothesis that asthma is one of the major causes of morbidity and mortality in HIV patients during antiretroviral therapy [61]. Recently, some studies have indicated that endometrial cancer has a favorable risk post-HIV infection [62]. One researcher found that some HIV drugs could inhibit in vitro bladder cancer migration and invasion [63]. In some clinical cases, HIV MDS transformed to acute myeloid leukemia [64]. A famous HIVpositive patient was healed of acute myeloid leukemia by allogeneic hematopoietic cell transplantation from a graft that carried the HIV-resistant CCR5 mutation [65]. The association between autoimmune thyroid disease and HIV infection has been suggested by several studies [66]. Although the HIV-related immunodeficiency state does not cause allograft rejection, anti-CD4 antibodies have considerable functions in the treatment of allograft rejection and the blockade of HIV infection [67, 68]. Recently, studies found that patients with HIV HAART-associated lipodystrophy syndrome had an increased risk of diabetes [69]. The impaired immune response to pneumococcal antigen pneumolysin due to HIV infection facilitates bacterial invasion [70]. Finally, HIV and AIDS are well-known subject diseases followed by malaria [71].

\section{Conclusion}

In this study, the correlation between ARGs and disease pathways genes expression were analyzed by CCA. The results showed that among ARGs, HLA-B, HLA-A, MH9, ZNED1, IRF1, TLR8, TSG101, NCOR2, and GML are the most significant genes correlated with other disease pathway genes. They are potential cross-links between AIDS and other diseases. Furthermore, gene pathways involved in several diseases such as asthma, autoimmune thyroid disease, and malaria were identified as an integrated pathway correlated with integrated ARGs. It suggests the risk of these diseases as AIDS complicating disease.

\section{Conflicts of Interest}

Authors declare no financial interest related to this work.

\section{Authors' Contributions}

Yahong Chen, Jinjin Yuan, and Xianlin Han contributed equally to this work.

\section{Acknowledgments}

This original study was supported by the Natural Science Foundation of Fujian Province (Grant nos. 2017J01157 and 2017J01158); the Scientific Foundation of Fuzhou City (Grant no. 2015-S-143-6); the Scientific Foundation of Fuzhou Health Department (Grant no. 2016-S-Wt7); and the Key Clinical Specialty Discipline Construction Program of Fuzhou, Fujian, China (Grant nos. 201510301 and 003040001).

\section{References}

[1] J. P. Zellweger, "HIV and tuberculosis: new perspectives for diagnosis and treatment," Revue Médicale Suisse, vol. 2, no. 87, pp. 2616-2619, 2006.

[2] A. E. Grulich, M. T. van Leeuwen, M. O. Falster, and C. M. Vajdic, "Incidence of cancers in people with HIV/AIDS compared with immunosuppressed transplant recipients: a meta-analysis," The Lancet, vol. 370, no. 9581, pp. 59-67, 2007.

[3] P. R. Kamerman, A. L. Wadley, and C. L. Cherry, "HIVassociated sensory neuropathy: risk factors and genetics," Current Pain and Headache Reports, vol. 16, no. 3, pp. 226-236, 2012.

[4] A. C. Hodowanec, A. Han, D. E. Barker, P. G. Rubinstein, and B. Max, "Thalidomide-associated thrombosis in the treatment of HIV-associated severe aphthous disease: a case report and review of the literature," Journal of the International Association of Physicians in AIDS Care, vol. 11, no. 6, pp. 345-347, 2012.

[5] M. Correale, G. A. Palmiotti, M. M. Lo Storto et al., "HIVassociated pulmonary arterial hypertension: from bedside to the future," European Journal of Clinical Investigation, vol. 45, no. 5, pp. 515-528, 2015.

[6] G. Dragovic, "Acute pancreatitis in HIV/AIDS patients: an issue of concern," Asian Pacific Journal of Tropical Biomedicine, vol. 3, no. 6, pp. 422-425, 2013.

[7] T. S. Uldrick and R. F. Little, "How I treat classical Hodgkin lymphoma in patients infected with human immunodeficiency virus," Blood, vol. 125, no. 8, pp. 1226-1235, 2015.

[8] F. Sbrana, M. Coceani, R. Iapoce, C. Petersen, and D. Rovai, "Rhabdomyolysis in an HIV-infected patient following coronary angiography: case report and literature review," International Journal of STD \& AIDS, vol. 25, no. 6, pp. 452-454, 2014.

[9] X. Shi, M. D. Sims, M. M. Hanna et al., "Neutropenia during HIV infection: adverse consequences and remedies," International Reviews of Immunology, vol. 33, no. 6, pp. 511-536, 2014.

[10] C. Chen, T. K. Hyun, X. Han et al., "Coexpression within integrated mitochondrial pathways reveals different networks in normal and chemically treated transcriptomes," International Journal of Genomics, vol. 2014, Article ID 452891, 10 pages, 2014. 
[11] C. Camilloni, A. B. Sahakyan, M. J. Holliday et al., "Cyclophilin A catalyzes proline isomerization by an electrostatic handle mechanism," Proceedings of the National Academy of Sciences of the United States of America, vol. 111, no. 28, pp. 10203-10208, 2014.

[12] J. Lu, Z. Han, Y. Liu et al., "A host-oriented inhibitor of Junin argentine hemorrhagic fever virus egress," Journal of Virology, vol. 88, no. 9, pp. 4736-4743, 2014.

[13] S. B. Kutluay, D. Perez-Caballero, and P. D. Bieniasz, "Fates of retroviral core components during unrestricted and TRIM5-restricted infection," PLoS Pathogens, vol. 9, no. 3, article e1003214, 2013.

[14] J. A. Estrada-Aguirre, I. Osuna-Ramírez, E. Prado Montes de Oca et al., "DEFB1 5'UTR polymorphisms modulate the risk of HIV-1 infection in Mexican women," Current HIV Research, vol. 12, no. 3, pp. 220-226, 2014.

[15] R. Srivastava, A. A. Khan, D. Spencer et al., "HLA-A02:01restricted epitopes identified from the herpes simplex virus tegument protein VP11/12 preferentially recall polyfunctional effector memory CD8+ $\mathrm{T}$ cells from seropositive asymptomatic individuals and protect humanized HLA-A 02:01 transgenic mice against ocular herpes," Journal of Immunology, vol. 194, no. 5, pp. 2232-2248, 2015.

[16] F. Zhu, P. Liu, J. Li, and Y. Zhang, "Eotaxin-1 promotes prostate cancer cell invasion via activation of the CCR3-ERK pathway and upregulation of MMP-3 expression," Oncology Reports, vol. 31, no. 5, pp. 2049-2054, 2014.

[17] A. J. Morgan, C. Guillen, F. A. Symon, S. S. Birring, J. J. Campbell, and A. J. Wardlaw, "CXCR6 identifies a putative population of retained human lung $\mathrm{T}$ cells characterised by co-expression of activation markers," Immunobiology, vol. 213, no. 7, pp. 599-608, 2008.

[18] R. H. Brakenhoff, M. Gerretsen, E. M. Knippels et al., "The human E48 antigen, highly homologous to the murine Ly-6 antigen ThB, is a GPI-anchored molecule apparently involved in keratinocyte cell-cell adhesion," The Journal of Cell Biology, vol. 129, no. 6, pp. 1677-1689, 1995.

[19] E. E. Wroblewski, P. J. Norman, L. A. Guethlein et al., "Signature patterns of MHC diversity in three Gombe communities of wild chimpanzees reflect fitness in reproduction and immune defense against SIVcpz," PLoS Biology, vol. 13, no. 5, article e1002144, 2015.

[20] B. J. Tallon, J. Bruneau, C. M. Tsoukas et al., "Time to seroconversion in HIV-exposed subjects carrying protective versus non protective KIR3DS1/L1 and HLA-B genotypes," PLoS One, vol. 9, no. 10, article e110480, 2014.

[21] E. M. Maloney, E. Pate, S. Z. Wiktor et al., "The relative distribution of $\mathrm{T}$ cell subsets is altered in Jamaican children infected with human T cell lymphotropic virus type I," The Journal of Infectious Diseases, vol. 172, no. 3, pp. 867-870, 1995.

[22] T. J. Lehky and S. Jacobson, "Induction of HLA class II in HTLV-I infected neuronal cell lines," Journal of Neurovirology, vol. 1, no. 2, pp. 145-156, 1995.

[23] F. Megret, C. Prehaud, M. Lafage et al., "Modulation of HLA-G and HLA-E expression in human neuronal cells after rabies virus or herpes virus simplex type 1 infections," Human Immunology, vol. 68, no. 4, pp. 294-302, 2007.

[24] E. Gazit, M. Sherf, E. Balbin, A. Muratov, I. Goldstein, and R. Loewenthal, "HLA-G expression is induced in Epstein-Barr virus-transformed B-cell lines by culture conditions," Human Immunology, vol. 68, no. 6, pp. 463-468, 2007.
[25] X. Chen, Y. Cai, and N. Sun, "The expression of HLA, Epstein-Barr virus nuclear antigen and the infiltration of T lymphocyte subsets in nasopharyngeal biopsy tissues," Zhonghua Er bi Yan Hou Ke Za Zhi, vol. 29, no. 1, pp. 6-9, 1994.

[26] S. Jeong, S. Park, B. W. Park, Y. Park, O. J. Kwon, and H. S. Kim, "Human leukocyte antigen-G (HLA-G) polymorphism and expression in breast cancer patients," PLoS One, vol. 9, no. 5, article e98284, 2014.

[27] N. D. Gladkova, A. A. Vostokova, K. V. Zvereva, L. G. Sibiriakova, and L. A. Pevnitskii, "The HLA system and Coxsackie B viral myocarditis in adults," Kardiologiia, vol. 26, no. 12, pp. 24-28, 1986.

[28] A. R. Hamzeh, P. Nair, and M. T. Al Ali, "The profile of HLADRB1 alleles in Arabs with type 1 diabetes; meta-analyses," Hla, vol. 87, no. 1, pp. 25-30, 2016.

[29] J. A. Traherne, W. Jiang, A. M. Valdes et al., "KIR haplotypes are associated with late-onset type 1 diabetes in EuropeanAmerican families," Genes and Immunity, vol. 17, no. 1, pp. 8-12, 2016.

[30] S. Morishima, K. Kashiwase, K. Matsuo et al., "High-risk HLA alleles for severe acute graft-versus-host disease and mortality in unrelated donor bone marrow transplantation," Haematologica, vol. 101, no. 4, pp. 491-498, 2016.

[31] E. M. Jacobson, H. Yang, F. Menconi et al., "Employing a recombinant HLA-DR3 expression system to dissect major histocompatibility complex II-thyroglobulin peptide dynamism: a genetic, biochemical, and reverse immunological perspective," The Journal of Biological Chemistry, vol. 284, no. 49, pp. 34231-34243, 2009.

[32] M. K. Misra, S. K. Pandey, R. Kapoor et al., "HLA-G gene expression influenced at allelic level in association with end stage renal disease and acute allograft rejection," Human Immunology, vol. 75, no. 8, pp. 833-839, 2014.

[33] T. Hays, V. D. D'Agati, J. A. Garellek et al., "Glomerular MYH9 expression is reduced by HIV-1," Aids, vol. 26, no. 7, pp. 797-803, 2012.

[34] E. Ballana, J. Senserrich, E. Pauls et al., "ZNRD1 (zinc ribbon domain-containing 1) is a host cellular factor that influences HIV-1 replication and disease progression," Clinical Infectious Diseases : An Official Publication of the Infectious Diseases Society of America, vol. 50, no. 7, pp. 1022-1032, 2010.

[35] C. C. Hardy, "Low-cost investments, mutual funds, and more," Dental Management, vol. 30, no. 2, pp. 55-57, 1990.

[36] J. Du, D. Zhang, W. Zhang et al., "pVHL negatively regulates antiviral signaling by targeting MAVS for proteasomal degradation," Journal of Immunology, vol. 195, no. 4, pp. 1782-1790, 2015.

[37] J. M. Cheng, M. R. Yao, Q. Zhu et al., "Silencing of stat4 gene inhibits cell proliferation and invasion of colorectal cancer cells," Journal of Biological Regulators and Homeostatic Agents, vol. 29, no. 1, pp. 85-92, 2015.

[38] T. Han, D. M. Xiang, W. Sun et al., "PTPN11/Shp2 overexpression enhances liver cancer progression and predicts poor prognosis of patients," Journal of Hepatology, vol. 63, no. 3, pp. 651-660, 2015.

[39] B. Iskender, K. Izgi, and H. Canatan, "Novel anti-cancer agent myrtucommulone-A and thymoquinone abrogate epithelialmesenchymal transition in cancer cells mainly through the inhibition of PI3K/AKT signalling axis," Molecular and Cellular Biochemistry, vol. 416, no. 1-2, pp. 71-84, 2016. 
[40] D. Guan and H. Y. Kao, "The function, regulation and therapeutic implications of the tumor suppressor protein, PML," Cell \& Bioscience, vol. 5, p. 60, 2015.

[41] X. Gu, B. H. Shin, Y. Akbarali et al., "Tel-2 is a novel transcriptional repressor related to the Ets factor Tel/ETV-6," The Journal of Biological Chemistry, vol. 276, no. 12, pp. 94219436, 2001.

[42] B. Huck, S. Duss, A. Hausser, and M. A. Olayioye, "Elevated protein kinase D3 (PKD3) expression supports proliferation of triple-negative breast cancer cells and contributes to mTORC1-S6K1 pathway activation," The Journal of Biological Chemistry, vol. 289, no. 6, pp. 3138-3147, 2014.

[43] C. G. Luder, C. Lang, M. Giraldo-Velasquez, M. Algner, J. Gerdes, and U. Gross, "Toxoplasma gondii inhibits MHC class II expression in neural antigen-presenting cells by downregulating the class II transactivator CIITA," Journal of Neuroimmunology, vol. 134, no. 1-2, pp. 12-24, 2003.

[44] P. J. Yan, L. Wang, X. L. Zha, and C. D. Lu, “Activating effect of hepatitis B virus preS/S protein on proliferating cell nuclear antigen gene promoter," Zhonghua Shi Yan He Lin Chuang Bing du Xue Za Zhi = Zhonghua shiyan He Linchuang Bingduxue Zazhi = Chinese Journal of Experimental and Clinical Virology, vol. 17, no. 1, pp. 42-45, 2003.

[45] Y. G. Yoo and M. O. Lee, "Hepatitis B virus X protein induces expression of Fas ligand gene through enhancing transcriptional activity of early growth response factor," The Journal of Biological Chemistry, vol. 279, no. 35, pp. 36242-36249, 2004.

[46] R. C. Su, A. Plesniarski, Z. Ao et al., "Reducing IRF-1 to levels observed in HESN subjects limits HIV replication, but not the extent of host immune activation," Molecular Therapy-Nucleic Acids, vol. 4, article e259, 2015.

[47] I. Fujimoto, T. Takizawa, Y. Ohba, and Y. Nakanishi, "Co-expression of Fas and Fas-ligand on the surface of influenza virus-infected cells," Cell Death and Differentiation, vol. 5, no. 5, pp. 426-431, 1998.

[48] X. Han, X. Li, S. C. Yue et al., "Epigenetic regulation of tumor necrosis factor alpha (TNFalpha) release in human macrophages by HIV-1 single-stranded RNA (ssRNA) is dependent on TLR8 signaling," The Journal of Biological Chemistry, vol. 287, no. 17, pp. 13778-13786, 2012.

[49] B. Willie, N. B. Hall, C. M. Stein et al., "Association of Toll-like receptor polymorphisms with HIV status in North Americans," Genes and Immunity, vol. 15, no. 8, pp. 569577, 2014.

[50] J. Ma, T. Chen, J. Mandelin et al., "Regulation of macrophage activation," Cellular and Molecular Life Sciences: CMLS, vol. 60, no. 11, pp. 2334-2346, 2003.

[51] N. Shaviya, V. Budambula, M. K. Webale, and T. Were, "Circulating interferon-gamma levels are associated with low body weight in newly diagnosed Kenyan non-substance using tuberculosis individuals," Inter Disciplinary Perspectives on Infectious Diseases, vol. 2016, Article ID 9415364, 9 pages, 2016.

[52] C. Chamontin, P. Rassam, M. Ferrer et al., "HIV-1 nucleocapsid and ESCRT-component Tsg101 interplay prevents HIV from turning into a DNA-containing virus," Nucleic Acids Research, vol. 43, no. 1, pp. 336-347, 2015.

[53] H. Chen, X. Liu, Z. Li, P. Zhan, and E. De Clercq, "TSG101: a novel anti-HIV-1 drug target," Current Medicinal Chemistry, vol. 17, no. 8, pp. 750-758, 2010.
[54] S. Shoji-Kawata, Q. Zhong, M. Kameoka et al., "The RING finger ubiquitin ligase RNF125/TRAC-1 down-modulates HIV-1 replication in primary human peripheral blood mononuclear cells," Virology, vol. 368, no. 1, pp. 191-204, 2007.

[55] P. Devaux, V. von Messling, W. Songsungthong, C. Springfeld, and R. Cattaneo, "Tyrosine 110 in the measles virus phosphoprotein is required to block STAT1 phosphorylation," Virology, vol. 360, no. 1, pp. 72-83, 2007.

[56] R. A. Fuentealba, Q. Liu, T. Kanekiyo, J. Zhang, and G. Bu, "Low density lipoprotein receptor-related protein 1 promotes anti-apoptotic signaling in neurons by activating Akt survival pathway," The Journal of Biological Chemistry, vol. 284, no. 49, pp. 34045-34053, 2009.

[57] S. Sil and T. Ghosh, "Cox-2 plays a vital role in the impaired anxiety like behavior in colchicine induced rat model of Alzheimer disease," Behavioural Neurology, vol. 2016, Article ID 1501527, 8 pages, 2016.

[58] C. Loeuillet, S. Deutsch, A. Ciuffi et al., "In vitro whole-genome analysis identifies a susceptibility locus for HIV-1," PLoS Biology, vol. 6, no. 2, article e32, 2008.

[59] T. Miyamura, T. Kanda, S. Nakamoto et al., "Hepatic STAT1-nuclear translocation and interleukin 28B polymorphisms predict treatment outcomes in hepatitis $\mathrm{C}$ virus genotype 1-infected patients," PLoS One, vol. 6, no. 12, article e28617, 2011.

[60] M. B. Drummond and G. D. Kirk, "HIV-associated obstructive lung diseases: insights and implications for the clinician," The Lancet Respiratory Medicine, vol. 2, no. 7, pp. 583-592, 2014.

[61] S. Gundavarapu, N. C. Mishra, S. P. Singh et al., "HIV gp120 induces mucus formation in human bronchial epithelial cells through CXCR4/alpha7-nicotinic acetylcholine receptors," PLoS One, vol. 8, no. 10, article e77160, 2013.

[62] I. Sivin and I. Batar, "State-of-the-art of non-hormonal methods of contraception: III. Intrauterine devices," The European Journal of Contraception \& Reproductive Health Care: The Official Journal of the European Society of Contraception, vol. 15, no. 2, pp. 96-112, 2010.

[63] M. Retz, S. S. Sidhu, J. Lehmann, H. Tamamura, N. Fujii, and C. Basbaum, "New HIV-drug inhibits in vitro bladder cancer migration and invasion," European Urology, vol. 48, no. 6, pp. 1025-1030, 2005.

[64] K. Takahashi, M. Yabe, I. Shapira, S. Pierce, G. Garcia-Manero, and M. Varma, "Clinical and cytogenetic characteristics of myelodysplastic syndrome in patients with HIV infection," Leukemia Research, vol. 36, no. 11, pp. 1376-1379, 2012.

[65] L. D. Petz, J. C. Burnett, H. Li et al., "Progress toward curing HIV infection with hematopoietic cell transplantation," Stem Cells and Cloning : Advances and Applications, vol. 8, pp. 109-116, 2015.

[66] L. G. Carvalho, F. Teixeira Pde, A. L. Panico et al., "Evaluation of thyroid function and autoimmunity in HIV-infected women," Arquivos Brasileiros de Endocrinologia e Metabologia, vol. 57, no. 1, pp. 450-456, 2013.

[67] J. E. Looney, A. Willinger, G. Lin, E. P. Rieber, G. Riethmuller, and J. Ghrayeb, "Expression and characterization of cM-T413, a chimeric anti-CD4 antibody with in vitro immunosuppressive activity," Journal of Immunotherapy with Emphasis on Tumor Immunology : Official Journal of the Society for Biological Therapy, vol. 16, no. 1, pp. 36-46, 1994.

[68] L. G. Carbone, D. J. Cohen, M. A. Hardy, A. I. Benvenisty, B. E. Scully, and G. B. Appel, "Determination of acquired 
immunodeficiency syndrome (AIDS) after renal transplantation," American Journal of Kidney Diseases: The Official Journal of the National Kidney Foundation, vol. 11, no. 5, pp. 387-392, 1988.

[69] S. Okada, M. Konishi, and H. Ishii, "Pioglitazone therapy for HIV/HAART-associated lipodystrophy syndrome could increase subcutaneous fat mass in non-lipoatrophic but not in lipoatrophic regions," BML Case Reports, vol. 25, 2016.

[70] B. M. Amdahl, J. B. Rubins, C. L. Daley, C. F. Gilks, P. C. Hopewell, and E. N. Janoff, "Impaired natural immunity to pneumolysin during human immunodeficiency virus infection in the United States and Africa," American Journal of Respiratory and Critical Care Medicine, vol. 152, no. 6, part 1, pp. 2000-2004, 1995.

[71] T. L. Drake, A. Devine, S. Yeung, N. P. Day, L. J. White, and Y. Lubell, "Dynamic transmission economic evaluation of Infectious disease interventions in low- and middleincome countries: a systematic literature review," Health Economics, vol. 25, Supplement 1, pp. 124-139, 2016. 

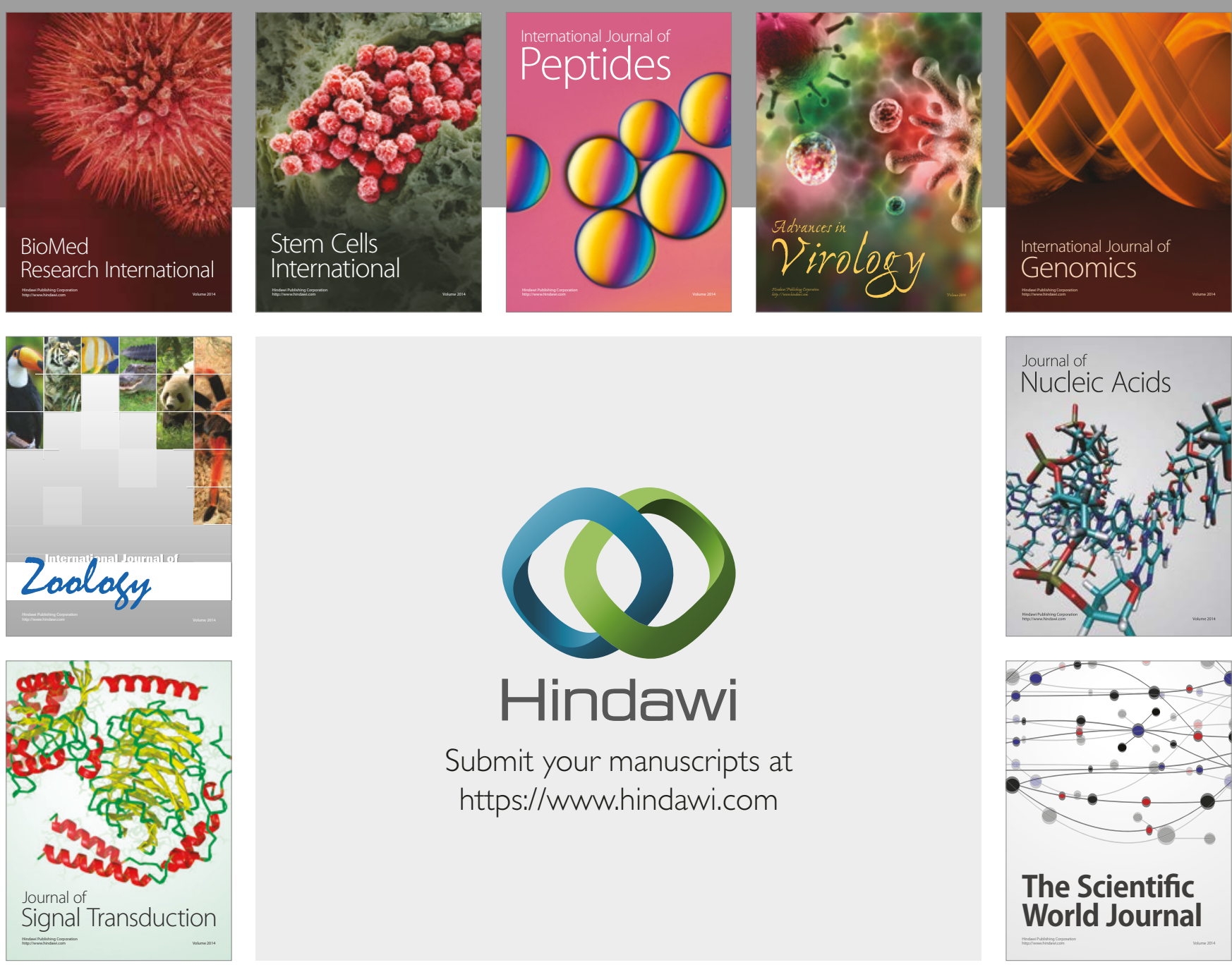

Submit your manuscripts at

https://www.hindawi.com
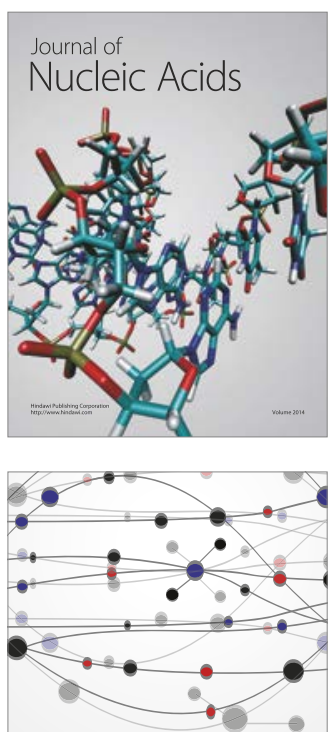

The Scientific World Journal

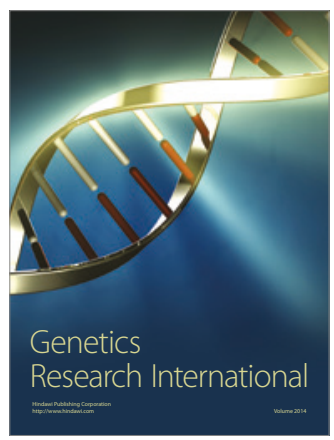

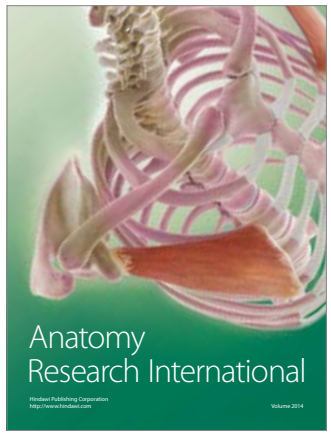

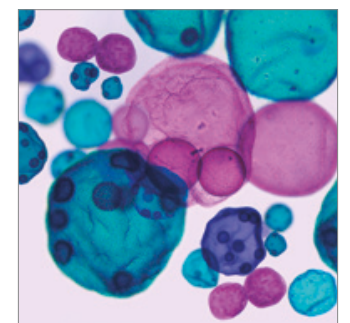

International Journal of Microbiology
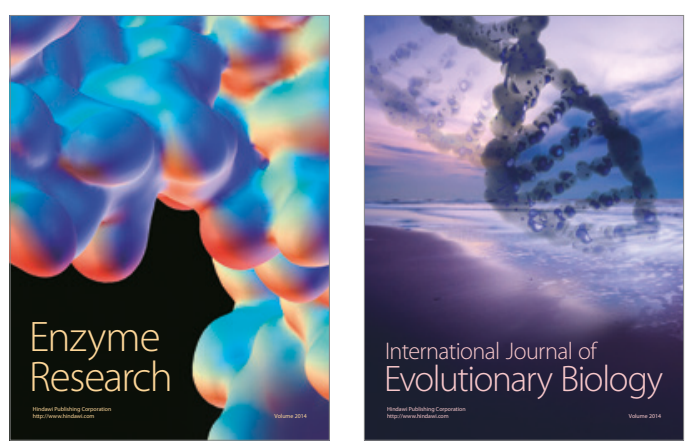
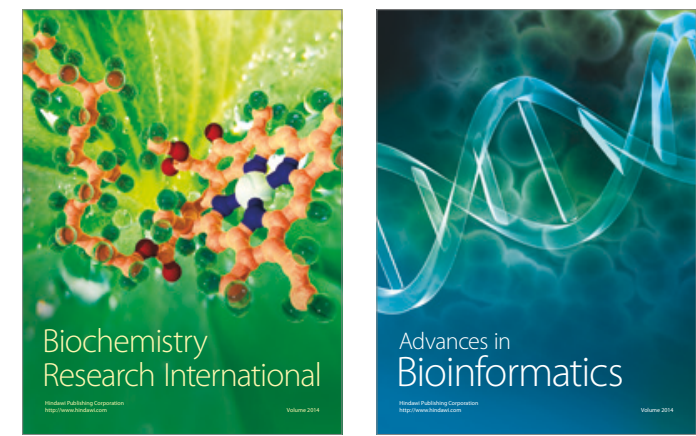

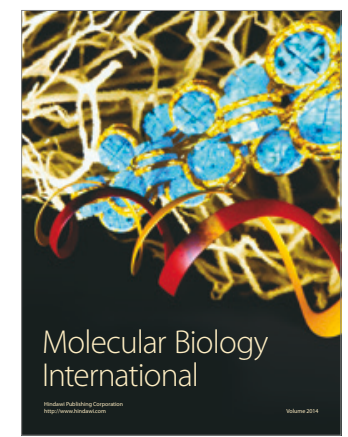

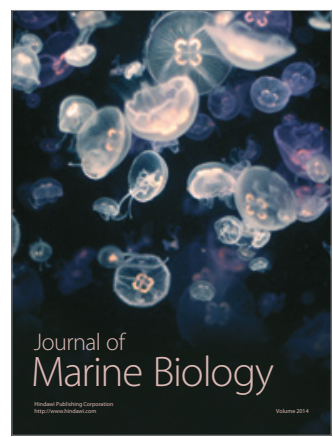

